RAPIDS

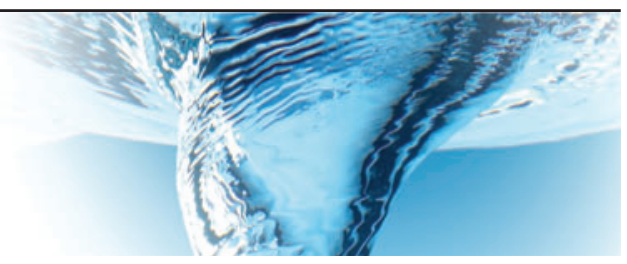

\title{
Length scale for the estimation of buzz frequency in the limit of high mechanical blockage in mixed-compression intakes
}

\author{
Manoj Kumar K. Devaraj ${ }^{1}$, Prahallada Jutur ${ }^{1}$, Srisha M.V. Rao ${ }^{1,}$, \\ Gopalan Jagadeesh ${ }^{1}$ and Ganesh T.K. Anavardham ${ }^{2}$ \\ ${ }^{1}$ Department of Aerospace Engineering, Indian Institute of Science, Bengaluru, 560055, India \\ ${ }^{2}$ Defence Research Development Laboratory, Hyderabad, 500058 Telangana, India
}

(Received 22 December 2020; revised 2 February 2021; accepted 10 March 2021)

\begin{abstract}
Oscillatory flow features are common in the unstart of hypersonic mixed-compression intakes and can be classified as low-amplitude or high-amplitude oscillatory unstarted flows. The low-amplitude oscillatory unstarted flow is driven by the shear layer from shock interactions ahead of the cowl, while the high-amplitude oscillatory unstarted flow is driven by the separation caused by shock-boundary-layer interaction on the ramp. While previous studies have observed these flow features and reported their associated frequency, there is no simple criterion available for predicting which mode will occur, and there is a lack of consensus on the appropriate frequency scaling parameter. We study a mixed-compression hypersonic intake in a hypersonic wind tunnel by varying the internal contraction ratio and the throttling ratio to observe various kinds of unstart regimes. Two significant conclusions emerge from considering the results for high-throttling-ratio conditions $(T R>0.55)$ from the current as well as previous studies. Firstly, the actual shock-on-lip condition at the cowl corresponding to the unthrottled condition, as observed from schlieren images, demarcates the boundary between the two modes of oscillatory unstart flows upon throttling. Secondly, a suitable length scale $\left(l^{*}\right)$, defined as the extent of the subsonic region in the unstarted flow (as observed from the experimental schlieren images), gives the appropriate frequency scaling parameter $\left(f^{*}=a_{0} / 4 l^{*}\right.$ where $a_{0}$ is the stagnation acoustic speed).
\end{abstract}

Key words: hypersonic flow, shock waves

$\dagger$ Email address for correspondence: srisharao@iisc.ac.in

(C) The Author(s), 2021. Published by Cambridge University Press. This is an Open Access article, distributed under the terms of the Creative Commons Attribution licence (http://creativecommons.org/ licenses/by/4.0/), which permits unrestricted re-use, distribution, and reproduction in any medium, provided the original work is properly cited. 


\section{Introduction}

Unstart is an undesirable phenomenon that may occur in high-speed intakes during which the shock system gets disgorged and may exhibit self-sustained oscillations (Wagner et al. 2009; Chang et al. 2017; Im \& Do 2018). These oscillations, known as 'buzz', have been reported and studied widely in supersonic intakes by various researchers (Ferri \& Nucci 1951; Dailey 1955; Sterbentz \& Davids 1955; Fisher, Neale \& Brooks 1970; Nagashima, Asanuma \& Obokata 1972; Van Wie, Kwok \& Walsh 1996; Trapier, Duveau \& Deck 2006; Soltani \& Sepahi-Younsi 2016). The majority of experiments on intake unstart simulate an increase in backpressure by using mechanical blockage at the end of the isolator to throttle the flow, and the resulting flow system resembles a forward-facing cavity (shock oscillations ahead of a forward-facing cavity were studied by Engblom et al. 1996); hence the quarter-wave resonator model ( $f=a_{0} / 4 l$, where $l$ is the geometric length between the cowl lip and the throat) has been proposed as a good model for scaling the frequency of the flow oscillations (Hankey \& Shang 1980). Furthermore, Newsome (1984) has also reported a similar expression to estimate the frequency of unstart based on average duct Mach number $(M)$, local speed of sound $(c)$ and characteristic length $(l)$, which is the distance between the cowl lip and the throat $\left(f=(2 n+1) c\left(1-M^{2}\right) / 4 l\right)$. The quarter-wave resonator model is a simple method and is able to estimate buzz frequency reasonably well in supersonic intakes. However, in hypersonic intakes, there is no consensus on the validity of this model. Tan, Shu \& Zhi-Long (2009) have used the model given by Newsome (1984) and have reported discrepancies with the experimentally observed frequencies in a hypersonic intake. On the other hand, recently, Berto et al. (2020) and the authors (Devaraj et al. 2020) have reported intake unstart at hypersonic Mach numbers, and have observed that the frequencies of oscillation are in reasonable agreement with the predictions based on the quarter-wave resonator model. The geometric differences between supersonic and hypersonic intakes - i.e. the fact that in the former, the length of the forebody is an order of magnitude lower than the distance between cowl lip and throat, while in the latter the two quantities are comparable (as shown in figure 1) - entail different flow physics. Most previous researchers have used the geometric length as the characteristic length scale, even for hypersonic intakes. We feel that the lack of consensus is mainly due to this extrapolation of the length scale from the supersonic intakes, and that a length scale based on the flow physics can extend the validity of the quarter-wave resonator model to hypersonic intakes as well.

Predicting the mode of unstart is as important as characterizing its frequency. In supersonic intakes, the flow oscillations (buzz) observed during unstart can be distinguished based on the flow features that drive these oscillations. Ferri \& Nucci (1951) have observed that the shock intersection gives rise to a vortex sheet, which separates the flow at the cowl surface upon impingement and initiates the buzz. In contrast, Dailey (1955) observed the buzz to be initiated by the ramp-side separation caused by the shock-boundary-layer interaction (SBLI). Fisher et al. (1970) report both types of instabilities at the same frequency but with significantly different amplitudes. Most of the studies on unstart in hypersonic intakes report oscillatory behaviour driven by ramp-side separation (Tan et al. 2009; Li et al. 2013). Zhang et al. (2016) have reported both types of oscillatory behaviour in a hypersonic mixed-compression intake at different Mach numbers. While the literature has distinguished the oscillatory flows based on the driving flow features, there is no simple criterion on the geometrical design or the operating parameters that distinguish between the two modes of unstart. In considering several previous experimental studies, we noticed that the location of the external shock system 
(a)

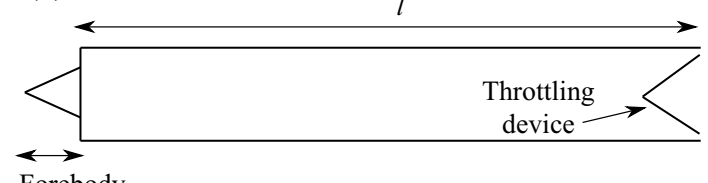

(b)

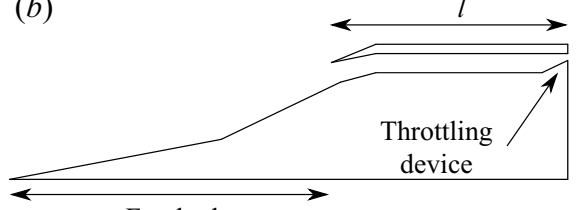

Forebody

Figure 1. Schematic depicting the geometrical aspects of (a) supersonic and (b) hypersonic unstart scenarios.

(a)

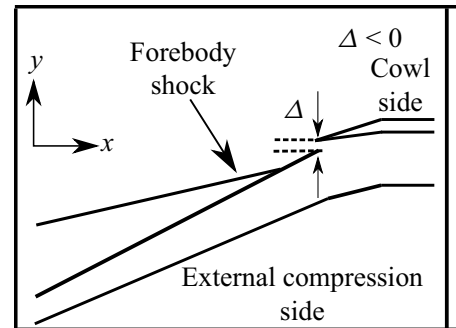

(b)

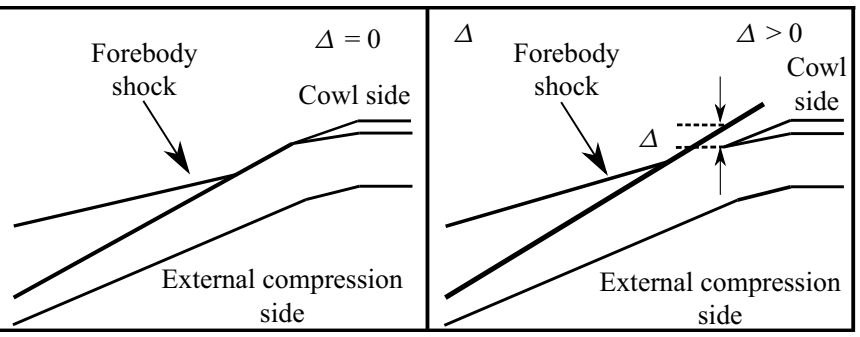

Figure 2. Schematic depicting different forebody/external compression shock impingement locations with respect to the cowl lip: (a) $\Delta<0,(b) \Delta=0$ and (c) $\Delta>0$, where $\Delta$ represents the normal distance (to the free-stream flow) between the external compression shock and the cowl lip, as observed from experimental schlieren images.

with respect to the cowl lip (shown in figure 2), as observed in schlieren images, has a significant bearing on the flow features during unstart. This aspect has not been given sufficient attention in previous studies.

Motivated by these two important observations, in the present work we carry out experiments to study unstart induced by throttling in a hypersonic mixed-compression intake at different internal contraction ratio $(I C R)$ conditions, which in turn correspond to different external compression shock locations with respect to the cowl lip. Our experiments aim to identify an appropriate length scale for frequency estimation at high throttling ratios (in non-reacting flows) and a criterion for distinguishing the mode of oscillatory unstart. These conditions mimic the high backpressure generated due to supersonic combustion and represent the worst-case scenario that can occur in real flight, where sustained oscillations may occur.

\section{Methodology}

The present work considers a mixed-compression intake consisting of external compression through two ramps at inclinations of $10.7^{\circ}$ and $25.6^{\circ}$ with the horizontal, and a constant-area isolator with a height $(h)$ of $6.2 \mathrm{~mm}$, as shown in figure 3 . The sidewalls in the isolator and the cowl region are provided with BK7 glass to enable optical access for schlieren. The intake model is equipped with a movable cowl as well as a flap located at the end of the isolator to independently vary the internal contraction ratio $(I C R)$ and throttling ratio $(T R)$, which are defined in $(2.1 a, b)$, where $A_{i s}, A_{t h}$ and $A_{i}$ represent the cross-sectional area of the isolator, throat and area at the cowl lip, respectively:

$$
I C R=\frac{A_{i}}{A_{i s}}, \quad T R=1-\frac{A_{t h}}{A_{i s}} .
$$




\section{M.K.K. Devaraj and others}

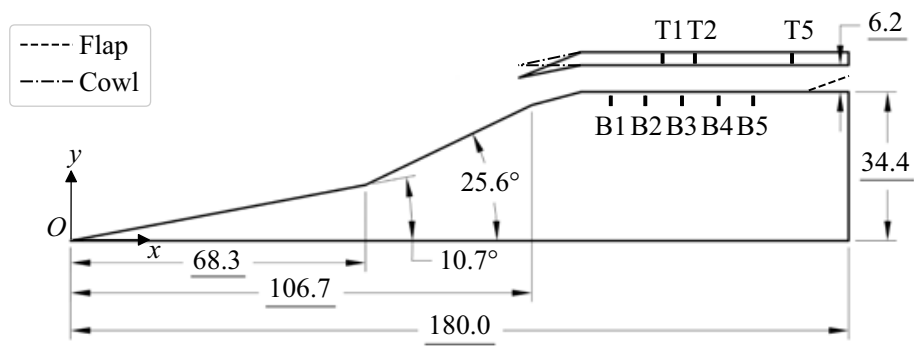

Figure 3. Schematic of the intake model indicating the movable cowl and flap. (All dimensions are in $\mathrm{mm}$.)

The experiments are conducted at a Mach number of $6 \pm 0.1$ in a hypersonic wind tunnel at the Indian Institute of Science. The facility is of blow-down type with air stored at high pressure on one end, driving the flow to vacuum on the other, providing a steady test time of $5 \mathrm{~s}$. The current experiments are carried out at a stagnation pressure of $7.55 \pm 0.04 \times$ $10^{5} \mathrm{~Pa}$ and a stagnation temperature of $423.80 \pm 9.47 \mathrm{~K}$. Accordingly, a flight-realistic Reynolds number of $8.73 \pm 0.19 \times 10^{6} \mathrm{~m}^{-1}$ has been achieved.

The experiments are conducted on the intake model by varying $T R$ during the steady test time, from a starting position of $T R=0$ to the final $T R$, for different $I C R$ conditions. Three different $I C R$ values, 1.19, 1.28 and 1.37, are considered. A nominal condition corresponding to a case without throttling $(T R=0)$ and a throttling condition at $T R=$ 0.56 are considered at all three $I C R$ values. In addition, a high $T R$ of 0.68 is also considered for the ICR values of 1.19 and 1.37 to investigate the effect of a higher $T R$ at different $I C R$ conditions. The intake model is instrumented with eight flush-mounted Kulite sensors, as shown in figure 3, capable of measuring unsteady pressure with an uncertainty of $2 \%$. The unsteady pressures from the Kulite sensors are acquired at a sampling rate of $100 \mathrm{kHz}$. Simultaneous schlieren visualization is also carried out for each experiment. A nano-pulsed laser is used as the light source (with $10 \mathrm{~ns}$ pulse duration) and is synchronized with the Photron SA4 high-speed camera to acquire images at $10000 \mathrm{fps}$. Further details of the facility and instrumentation are given in Devaraj et al. (2020). The dynamic content associated with the flow is analysed with the help of fast Fourier transform (FFT) of the unsteady pressure signals as well as dynamic mode decomposition (DMD) of the schlieren image sequence. The methodology used for the DMD analysis is given in Rao \& Karthick (2019).

\section{Results}

\subsection{Started flows}

The typical flow features observed during a started flow through the intake are presented in figure 4, which corresponds to the nominal condition of $T R=0$ at different $I C R$ values. Two oblique shocks, OS1 and OS2, emanate from the external compression ramps. The flow turns back into the isolator through cowl shocks CS1 and CS2 and remains supersonic through the isolator region across all three ICR conditions. The change in ICR leads to variation in the strength of the cowl shocks CS1 and CS2, and accordingly, differences are observed in the isolator flowfield. Figure $4(d)$ shows the non-dimensional pressure profile along the isolator for different $I C R$ conditions. Peak pressures of $\approx 50$ times the free-stream pressure in the isolator are observed at $I C R=1.19$ and $I C R=1.28$, while the same at $I C R=1.37$ is $\approx 60$ times the free-stream pressure. It is important to note that a 
(a)

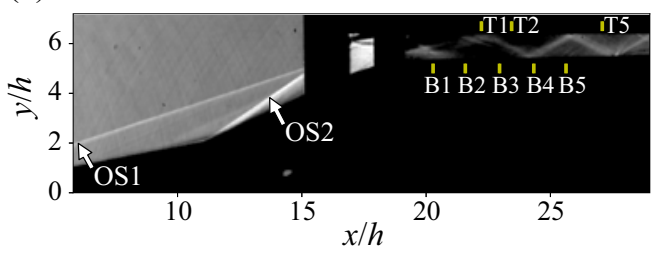

(b)

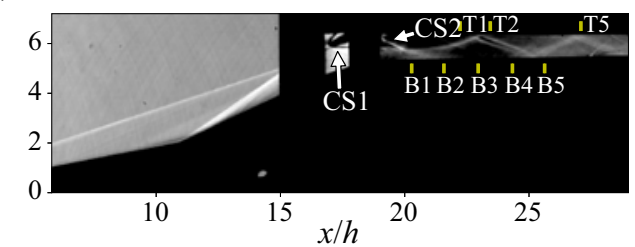

(d)
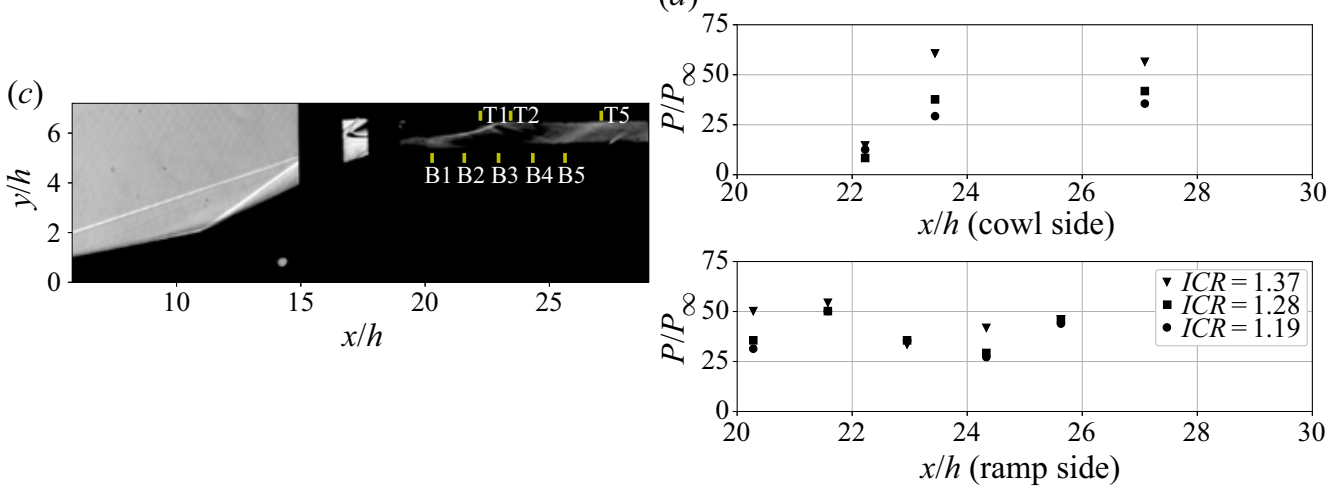

Figure 4. Time-averaged schlieren images at $(a) I C R=1.37$, (b) $I C R=1.27$ and (c) $I C R=1.19$, showing the started flow through the intake. $(d)$ Pressure profile along the isolator for different $I C R$ conditions that correspond to started flow in the intake.

variation in $I C R$ leads to a change in impingement location of the external compression shock on the cowl surface. At $I C R=1.37$ and $I C R=1.28$, the external compression shock impinges on the cowl surface, while it misses the cowl lip for $I C R=1.19$.

\subsection{Unstarted flows}

The flow features of the intake are modified significantly with the introduction of throttling. For the scope of the present work, the focus is on the final flow features after achieving the desired throttling. In the present study, unstarted flows are observed at $T R \geq 0.56$ for all three $I C R$ cases. The time-averaged schlieren images corresponding to $I C R$ cases of 1.37 and 1.28 at $T R=0.56$ are shown in figure 5. A detached cowl shock (DCS) with a type V shock interaction, forming a supersonic jet SJ that impinges onto the ramp side and terminates very close to the cowl leading edge, can be observed. The movement of this shock system ahead of the cowl is not significant, and hence a time-averaged image is presented. The flow structure comprising the DCS and SJ is observed farther downstream at $I C R=1.28$ than at $I C R=1.37$. The FFT of the pressure signal and DMD of the schlieren visualizations do not show any dominant frequencies associated with the flow features (see figure $6 a$ ) for $T R=0.56$ at $I C R=1.37$, with a peak pressure of approximately 200 times the free-stream pressure. Similar behaviour is observed for the case of $T R=0.56$ and $I C R=1.28$, which is not shown here. However, at a higher $T R$ of 0.68 , a dominant peak at $3686 \mathrm{~Hz}$ corresponding to a low-amplitude oscillatory unstarted flow is observed in both the FFT of the pressure signal and the DMD of the schlieren images. However, the flow features are not altered significantly compared to those of $T R=0.56$. 

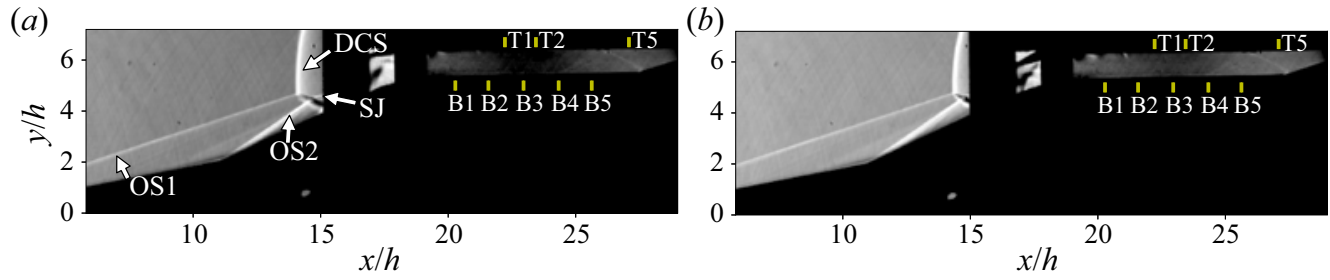

Figure 5. Time-averaged schlieren images corresponding to $T R=0.56$ at $(a) I C R=1.37$ (see supplementary movie 1, available at https://doi.org/10.1017/jfm.2021.230) and (b) $I C R=1.28$.

(a)
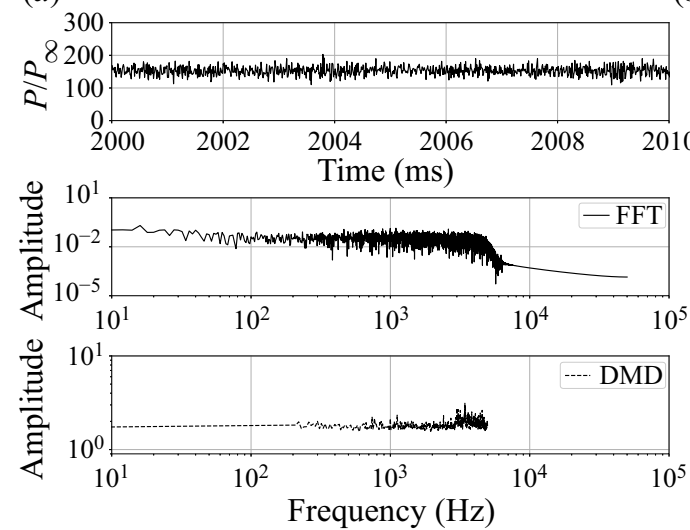

(b)
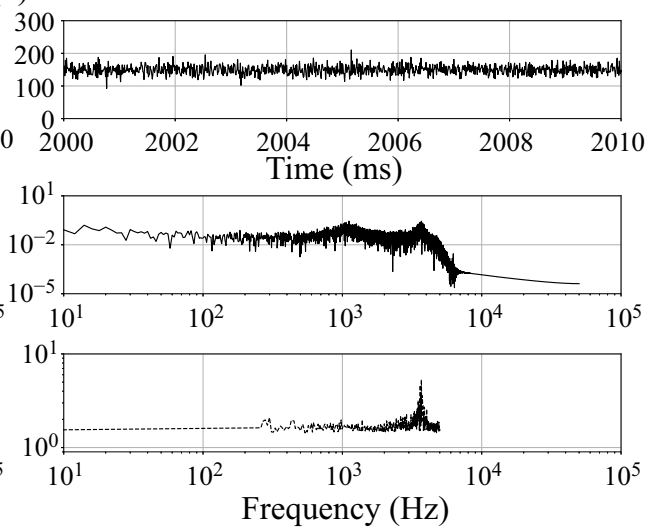

Figure 6. Variation of pressure with time, corresponding FFT of the B5 signal and DMD of the schlieren visualizations, in the case $I C R=1.37$, with $(a) T R=0.56$ and $(b) T R=0.68$.

In contrast, at545 ICR $=1.19$, high-amplitude oscillatory unstarted flow is observed for $T R \geq 0.56$. Figure 7 presents the sequence of instantaneous schlieren images at $T R=0.56$ for this $I C R$. The flow features in common with $I C R \geq 1.28$ are OS1, OS2, DCS and SJ. The two main differences observed at this $I C R$ compared to the other cases are that $(a)$ the shock system is being pulled downstream closer to the cowl leading edge, and $(b)$ the ramp-side separation bubble (SLE) is seen upstream of the point of shock interaction (at $x / h=14$ ). The frame corresponding to the most upstream location of the expelled shock system is taken as the reference time $t_{\text {ref }}$ for this case. At $t=t_{\text {ref }}+0.4 \mathrm{~ms}$, the shock system has moved downstream, behind the sidewall, and SJ is seen near the cowl leading edge and enters the isolator. In this instance, a high-pressure region is created near the exit of the isolator, and an upstream motion of the shock system is initiated. The subsequent frames corresponding to $t=t_{\text {ref }}+0.8$ and $t_{r e f}+1$ ms show the upstream movement of the flow, thereby completing the cycle. A plot of the pressure signal at sensor location B5, the corresponding FFT of the pressure signal and the DMD of the schlieren images are shown in figure 8. A dominant peak is observed at a frequency of $950 \mathrm{~Hz}$ at $T R=0.56$. On the other hand, $T R=0.68$ reveals a dominant peak at $1100 \mathrm{~Hz}$ with an increase in the amplitude of pressure oscillation. In both these cases, peak pressures of about 200 times the free-stream pressure are observed, with high-amplitude oscillation in comparison to the case $I C R=1.37$ (figure 6). However, the flow features corresponding to this higher-TR case, which were already reported in Devaraj et al. (2020), are similar to those of $T R=0.56$. At $I C R=1.19$, the separation bubble present on the ramp side deflects 

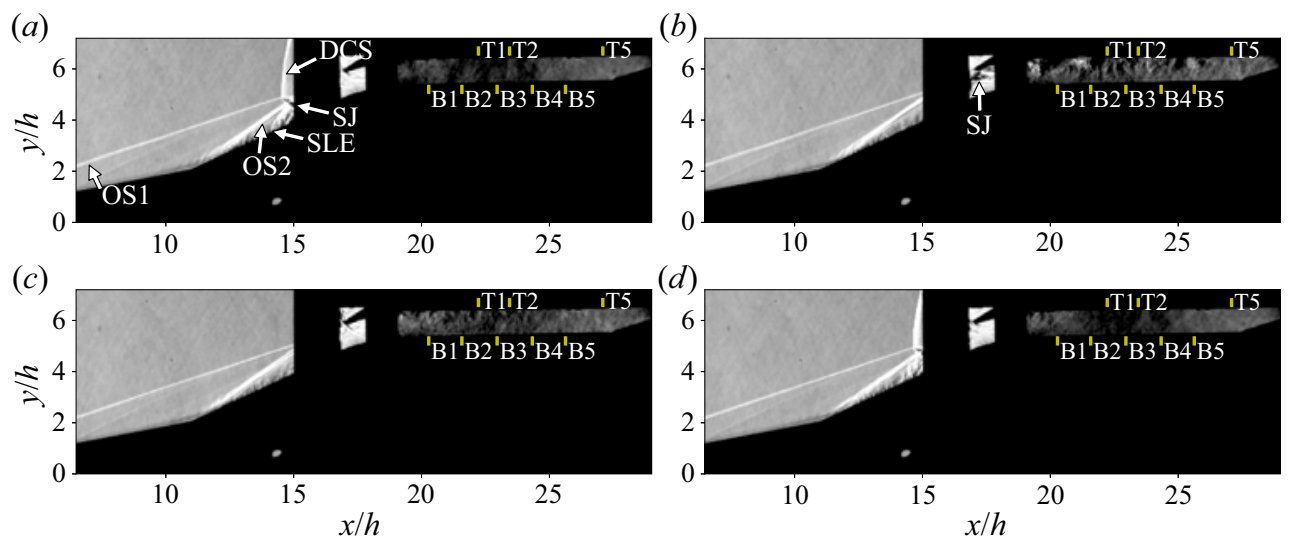

Figure 7. Sequence of events observed for $T R=0.56$ at $I C R=1.19:(a) t_{\text {ref }}$, (b) $t_{\text {ref }}+0.4 \mathrm{~ms}$, (c) $t_{\text {ref }}+0.8$ $\mathrm{ms}$ and $(d) t_{\text {ref }}+1 \mathrm{~ms}$ (see supplementary movie 2 ).

(a)
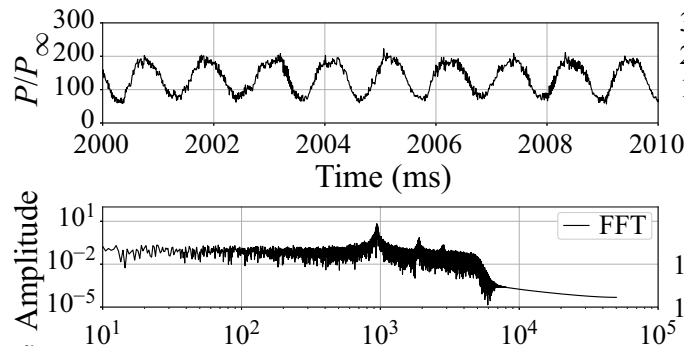

(b)
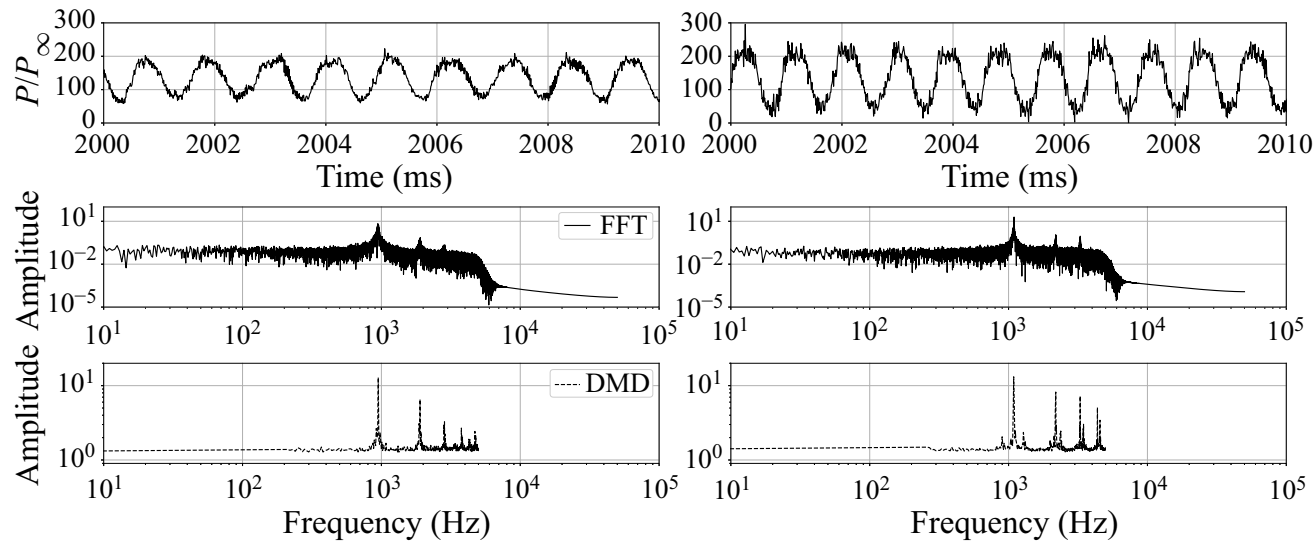

Figure 8. Variation of pressure with time, corresponding FFT of the B5 signal and DMD of the schlieren visualizations, in the case $I C R=1.19$, with $(a) T R=0.56$ and $(b) T R=0.68$.

SJ away from the ramp wall and allows it to enter the isolator. The entrainment observed at $I C R=1.19$ is driven by the ramp-side separation, similarly to the observations made by Dailey (1955); correspondingly, the amplitude of the oscillations is also found to be relatively high. The low-amplitude oscillations observed at $I C R=1.37$ are characteristic of the mechanism proposed by Ferri \& Nucci (1951). According to Ferri \& Nucci (1951), the shear layer emanating from the point of the shock interaction is responsible for the instabilities during the unstart.

\subsection{A criterion distinguishing between the two modes of unstart}

Figure $9(a)$ shows a plot representing the higher-throttling-ratio conditions considered in the present work within the purview of Kantrowitz and isentropic limits of intake operation. The Mach number at the entrance of the internal compression section during the started operation $\left(M_{i}\right)$ for various $I C R$ conditions is calculated based on two-dimensional Reynolds-averaged Navier-Stokes simulations using the open-source suite SU2 Falcon 

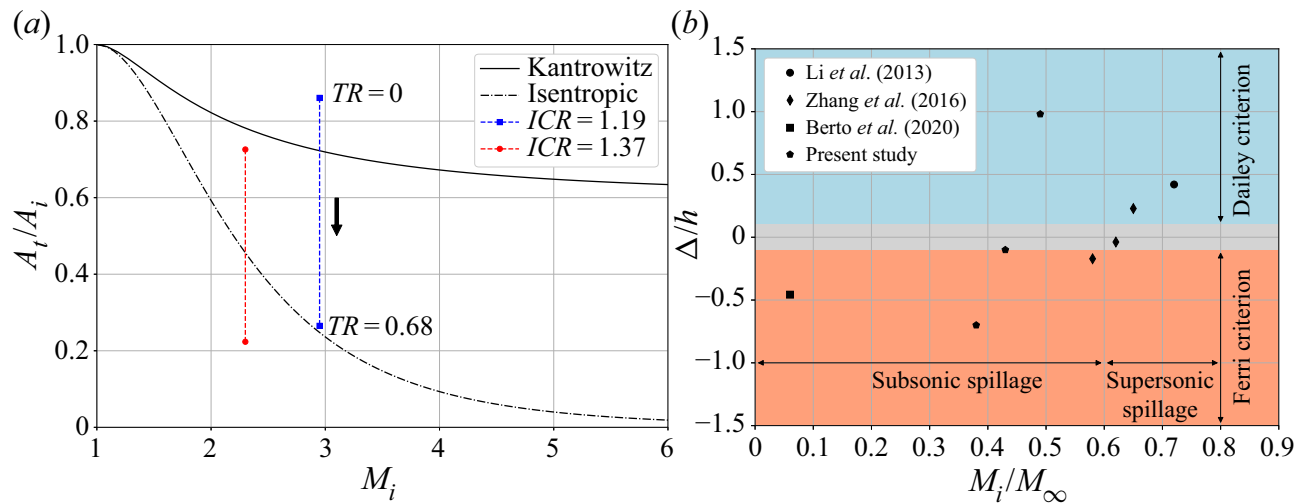

Figure 9. (a) A plot representing the higher-TR conditions in the purview of Kantrowitz and isentropic limits. (b) Comparison of non-dimensional location of shock impingement with normalized Mach number from various studies, including the present one.

(Palacios et al. 2013). At $I C R=1.19$, the forebody shock misses the cowl. An increase in $I C R$ corresponds to the impingement of the forebody shock onto the cowl surface, thereby reducing $M_{i}$ for the case of $I C R=1.37$. Because of this reduction in $M_{i}$ for the same throttling ratio $(T R=0.68)$, the $I C R=1.37$ falls below the isentropic limit, while the same for $I C R=1.19$ is above the isentropic limit. Consequently, high- and low-amplitude oscillatory unstarted flows are observed at $I C R=1.19$ and $I C R=1.37$, respectively. Figure $9(b)$ presents a comparison of the non-dimensional location of shock impingement with free-stream Mach number from various studies, including the present one, where the effect of throttling has led to unstart. The $\Delta / h$ values are obtained from the schlieren images reported in the respective studies, with $\Delta$ denoting the distance of the shock impingement from the cowl lip in the direction normal to the free-stream flow during the started operation. When $\Delta / h>0$ (the shock misses the cowl), the boundary-layer separation at the ramp side is identified as the driving flow feature (Dailey criterion), and a high-amplitude oscillatory unstart is observed. High-amplitude oscillatory unstarts have been reported by Tan et al. (2009) and Li et al. (2013), where the intake operates with $\Delta / h>0$. On the other hand, the shear layer emanating from the point of shock interaction is observed to be the driving flow feature (Ferri criterion) for the low-amplitude oscillatory unstart when $\Delta / h<0$ (the shock impinges on the cowl surface). From figure $9(b)$, it is evident that $\Delta / h=0$, which corresponds to shock-on-lip (SOL) condition during the started operation, is the boundary between the Dailey and Ferri criteria for hypersonic mixed-compression intakes operating with free-stream Mach numbers between 5 and 7 . It is important to note that SOL here corresponds to that observed from the experimental schlieren images. The actual SOL criterion, therefore, can be useful in identifying the driving flow features and type of oscillations that can occur during unstart. The appendix gives additional supporting evidence to emphasize that the actual SOL is the distinguishing criterion.

\subsection{Buzz frequency scaling}

In our previous work (Devaraj et al. 2020), we have shown that the flow features in the intake differ significantly between static and dynamic flap experiments in lower- $T R$ cases. For example, the intake remains in the started condition in a dynamic flap experiment, 
(a)
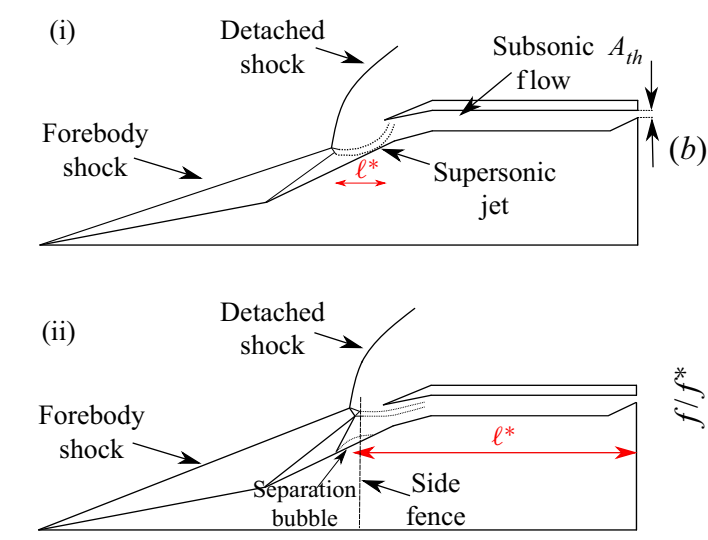

(b)
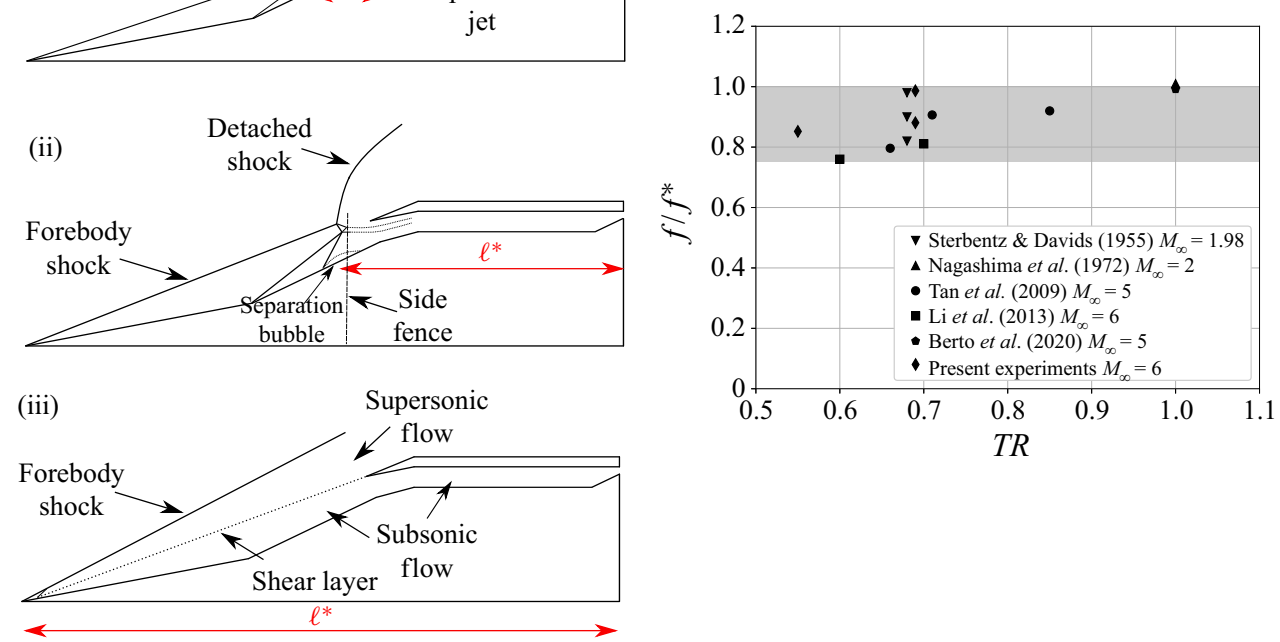

Figure 10. (a) Schematic of flow features observed during unstart, clearly marking the appropriate length scale that needs to be considered for frequency estimation based on the quarter-wave resonator model where the flow oscillations are caused by (i) ramp-side separation (Dailey criterion) and subsonic spillage, (ii) cowl-side separation (Ferri criterion) and subsonic spillage, and (iii) ramp-side separation and supersonic spillage (Tan et al. 2009; Li et al. 2013). (b) A plot of frequency normalized with the frequency $(f)$ estimated using the quarter-wave resonator $\left(f^{*}=a_{0} / 4 l^{*}\right)$, as a function of $T R$ for $T R>0.5$.

while it is unstarted during a static flap experiment at $T R=0.3$. Moreover, in a flight scenario, the combustion process is initiated after the establishment of the started flow through the intake. Hence, a dynamic throttling would closely mimic the practical scenario at lower- $T R$ conditions, where there is a difference between static and dynamic flap experiments. On the other hand, the oscillatory unstarted flows observed at higher $T R$ are independent of the mode of flap operation. In addition, Tan et al. (2009) and Li et al. (2013) have shown that the frequency remains relatively unchanged beyond a particular throttling ratio. Hence, a scaling analysis for the limiting case of $T R \rightarrow 1$ will be applicable independent of the mode of flap operation.

It is evident from the FFT and DMD plots that the unstarted flow exhibits self-sustained oscillations at a well-defined dominant frequency at $T R=0.68$. In these high- $T R$ conditions $(T R \rightarrow 1)$, the blockage imposed by the flap gives rise to a configuration similar to that of a forward-facing cavity; accordingly, we propose a suitable length scale $\left(l^{*}\right)$ that is defined as the extent of the subsonic region in the unstarted flow. In the present study, during low-amplitude oscillatory unstarted flow, the shear layer emanating from the point of shock interaction terminates near the cowl to form a virtual forward-facing cavity. Hence, the distance between the point of shock interaction and the impingement of the shear layer on to the cowl surface is an appropriate length scale (figure 10a(i)). On the other hand, during the high-amplitude oscillatory unstarted flow, the appropriate length scale would be the distance between the leading edge of the separation bubble and the throat, which is formed at the exit of the isolator (figure 10a(ii)). With this choice 


\begin{tabular}{|c|c|c|c|c|c|c|c|c|}
\hline & \multirow{2}{*}{$M_{i} / M_{\infty}$} & \multirow[t]{2}{*}{ Type of spillage } & \multirow[t]{2}{*}{$\Delta / h$} & \multirow[t]{2}{*}{$\begin{array}{l}\text { Driving flow } \\
\text { feature of } \\
\text { oscillation }\end{array}$} & \multirow[t]{2}{*}{$a_{0}\left(\mathrm{~m} \mathrm{~s}^{-1}\right)$} & \multirow[t]{2}{*}{$l^{*}(\mathrm{~m})$} & \multicolumn{2}{|c|}{ Frequency $(\mathrm{Hz})$} \\
\hline & & & & & & & Experiment & Theory \\
\hline Berto et al. (2020) & 0.06 & subsonic & - & SL & 395.9 & 0.070 & 1423 & 1411 \\
\hline Present study & 0.38 & subsonic & - & SL & 410.2 & 0.025 & 3686 & 4183.55 \\
\hline Present study & 0.49 & subsonic & + & RSSB & 410.2 & 0.092 & 1100 & 1115.6 \\
\hline Tan et al. (2009) & 0.65 & supersonic & + & RSSB & 482.8 & 0.325 & 337 & 371.79 \\
\hline Li et al. (2013) & 0.72 & supersonic & + & RSSB & 570.8 & 0.289 & 400 & 493.9 \\
\hline
\end{tabular}

Table 1. Details of normalized Mach number at the entry to the internal contraction section $\left(M_{i} / M_{\infty}\right)$, normalized distance of the forebody shock impingement location from the cowl lip, driving flow feature (SL - shear layer; RSSB - ramp-side separation bubble), type of spillage and a comparison of frequency values observed during experiments and the theoretical estimate $\left(a_{0} / 4 l^{*}\right)$, along with the individual values of $a_{0}$ and $l^{*}$, from different experimental studies pertaining to hypersonic intakes.

of the appropriate length scale in each of these cases, the frequency estimated using the quarter-wave resonator model matches the experimental values reasonably well. To further strengthen the validity of this scaling, table 1 compares the frequency values at the highest $T R$ from different experimental studies of mixed-compression intakes against theoretical estimates. For intakes that exhibit unstart with supersonic spillage, the separation region extends up to the leading edge of the forebody, with subsonic flow prevailing over the ramp side as well as the isolator (Tan et al. 2009; Li et al. 2013). Hence the appropriate length scale in this case would be the axial distance between the throat and the leading edge of the intake (figure 10a(iii)). Figure $10(b)$ represents the experimentally observed frequency values normalized with theoretical estimates for different $T R(T R>0.5)$ over a wide range of Mach numbers (ranging from $M_{\infty}=2$ to $M_{\infty}=6$ ), from both previous literature and the present study. It is interesting to note that the assumption of similarity to a forward-facing cavity leads to reasonably accurate frequency estimates (with a maximum difference of $\approx 20 \%$ ), even for $T R$ values as low as $T R=0.55$. It may be noted that some researchers (Dailey 1955; Rodi, Emami \& Trexler 1996; Trapier et al. 2006) have reported higher harmonics of the fundamental mode $\left(f=a_{0} / 4 l\right)$ to be dominant in supersonic intakes, the reasons for which are unclear. Furthermore, when unstart is induced by combustion heat release (as in Im et al. 2016), chemical reactions may play a significant role, and identifying the exact length scale may be difficult. Detailed studies using experiments and high-fidelity numerical simulations are required to clarify the dynamics of shock oscillations in the context of chemically reacting flows.

\section{Conclusion}

Collating data corresponding to high-throttling-ratio cases from the current and previous studies pertaining to mixed-compression intakes, two modes of unstart can be identified. High-amplitude oscillatory unstarted flow is driven by the separation bubble on the ramp side (Dailey criterion). Low-amplitude oscillatory unstarted flow is driven by the shear layer emanating from the shock interactions (Ferri criterion). The two significant conclusions of this work are as follows: $(a)$ the actual SOL (corresponding to the unthrottled condition) is the demarcating boundary between the two modes of oscillatory unstart observed upon high throttling; $(b)$ the extent of the subsonic region (as observed 
(a)

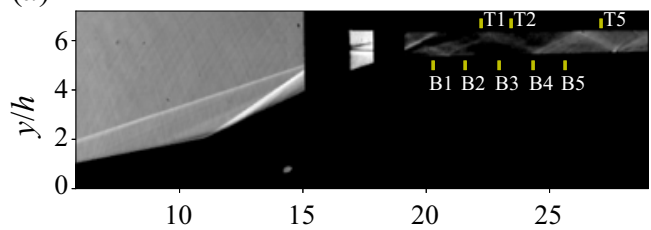

(c)

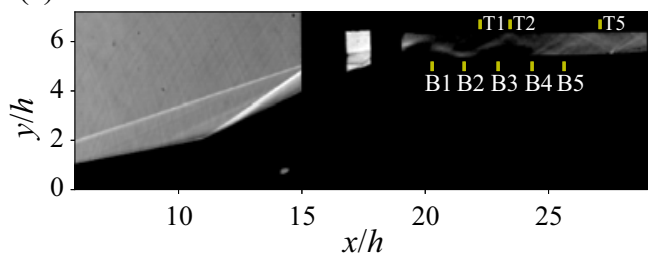

(b)

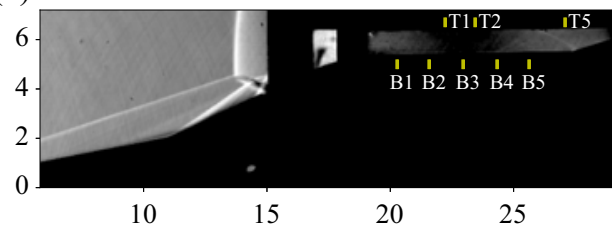

$(d)$

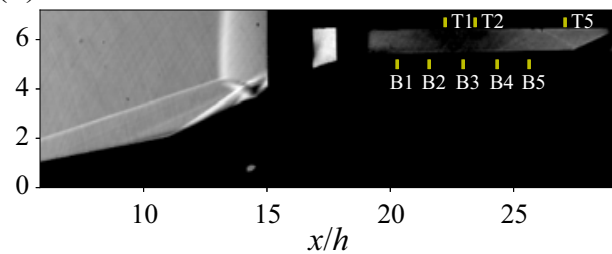

Figure 11. Time-averaged schlieren images for the cases corresponding to $(a) I C R=1.61, T R=0$, (b) $I C R=1.61, T R=0.56$, (c) $I C R=1.85, T R=0$ and $(d) I C R=1.85, T R=0.75$.

(a)
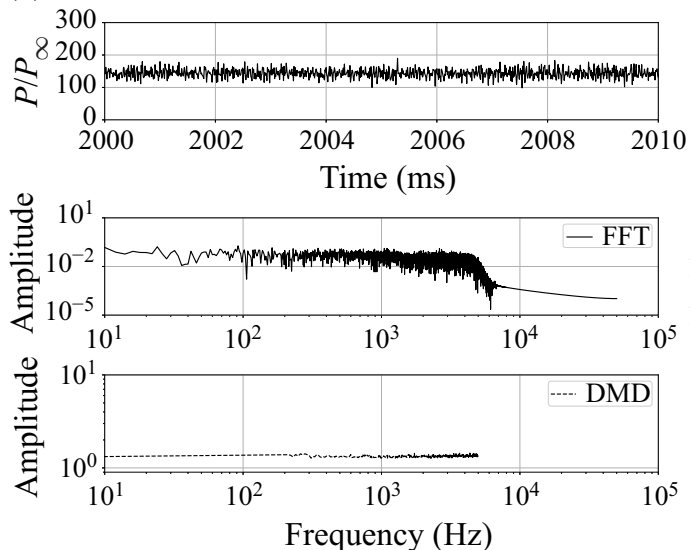

(b)
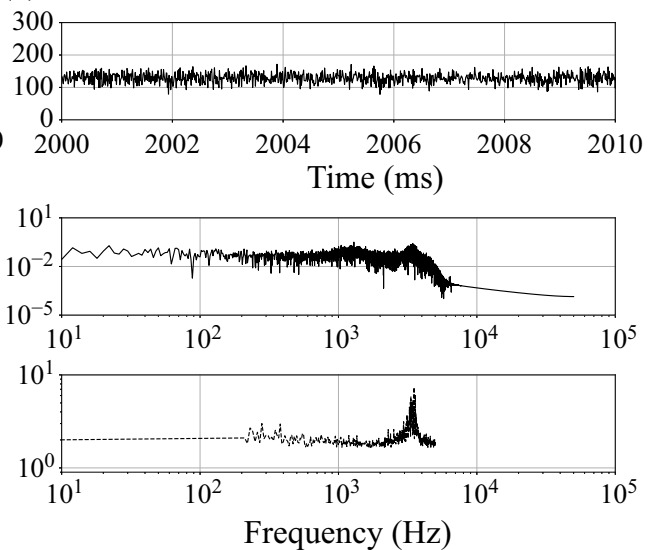

Figure 12. Variation of pressure with time, corresponding FFT of the B5 signal and DMD of the schlieren visualizations, in the cases $(a) I C R=1.61, T R=0.56$ and $(b) I C R=1.85, T R=0.75$.

from the experimental schlieren images) is the appropriate length scale to be used in the quarter-wave resonator model.

Supplementary movies. Supplementary movies are available at https://doi.org/10.1017/jfm.2021.230.

Acknowledgements. The authors would like to thank BrahMos Aerospace Private Limited, New Delhi, and an Indian Institute of Science Startup Grant for funding this research work. The authors would also like to thank Shivashankar and Shantha Kumar for their help in operating the $0.5 \mathrm{~m}$ Hypersonic Wind Tunnel, Murthy and Raju for their help in fabrication, Parthiban (Novatek Automation) for designing the mechanism of the flap and the cowl, Ramji V. for his help in setting up the apparatus for schlieren visualization and members of the Laboratory for Hypersonic and Shockwave Research at the Indian Institute of Science. The first author would like to acknowledge discussions with A. Bajpai and Dr T. Singh.

Declaration of interests. The authors report no conflict of interest. 


\title{
M.K.K. Devaraj and others
}

\author{
Author ORCIDs. \\ (1) Prahallada Jutur https://orcid.org/0000-0001-7826-2770; \\ Srisha M.V. Rao https://orcid.org/0000-0002-6375-7137; \\ Dopalan Jagadeesh https://orcid.org/0000-0002-5495-9351.
}

\section{Appendix}

To further emphasize that the actual SOL is the demarcating criterion for the two modes of unstart, additional evidence corresponding to representative high- $T R$ cases at $I C R=1.61$ and $I C R=1.85$ is shown in figures 11 and 12 , which correspond to $\Delta / h<0$. For these $I C R$ cases, the flow features in the unthrottled conditions, as shown in figure $11(a, c)$, are significantly different from those of the cases shown in $\$ 3.1$. However, the flow features upon throttling (figure $11 b, d$ ) are similar to those shown in figure 5. Moreover, the magnitude of pressure and the dynamic content as seen in figure 12 are similar to those presented in figure 6.

\section{REFERENCES}

Berto, F., Benini, E., Wyatt, C. \& Quinn, M.K. 2020 Time-accurate experimental investigation of hypersonic inlet buzz at Mach 5. AIAA J. 58 (5), 2197-2205.

Chang, J., LI, N., XU, K., BAO, W. \& YU, D. 2017 Recent research progress on unstart mechanism, detection and control of hypersonic inlet. Prog. Aerosp. Sci. 89, 1-22.

DAILEY, C.L. 1955 Supersonic diffuser instability. J. Aeronaut. Sci. 22 (11), 733-749.

Devaraj, M.K.K., Jutur, P., RaO, S.M.V., Jagadeesh, G. \& Anavardham, G.T.K. 2020 Experimental investigation of unstart dynamics driven by subsonic spillage in a hypersonic scramjet intake at Mach 6. Phys. Fluids 32 (2), 026103.

Engblom, W.A., Yuceil, B., Goldstein, D.B. \& Dolling, D.S. 1996 Experimental and numerical study of hypersonic forward-facing cavity flow. J. Spacecr. Rockets 33 (3), 353-359.

FERRI, A. \& NUCCI, L.M. 1951 The origin of aerodynamic instability of supersonic inlets at subcritical condition. NACA RM L50K30, pp. 1-111.

Fisher, S.A., NeAle, M.C. \& BROOKS, A.J. 1970 On the Sub-Critical Stability of Variable Ramp Intakes at Mach Numbers Around 2. HM Stationery Office Richmond.

HANKey, W. \& Shang, J. 1980 Analysis of self-excited oscillations in fluid flows. In 13th Fluid and PlasmaDynamics Conference, p. 1346.

Im, S., Baccarella, D., McGann, B., Liu, Q., Wermer, L. \& Do, H. 2016 Unstart phenomena induced by mass addition and heat release in a model scramjet. J. Fluid Mech. 797, 604.

IM, S.-K. \& Do, H. 2018 Unstart phenomena induced by flow choking in scramjet inlet-isolators. Prog. Aerosp. Sci. 97, 1-21.

LI, Z., GAO, W., JANG, H. \& YANG, J. 2013 Unsteady behaviors of a hypersonic inlet caused by throttling in shock tunnel. AIAA J. 51, 1-22.

Nagashima, T., Asanuma, T. \& Oвокаta, T. 1972 Experiment of Supersonic Air Intake Buzz. Institute of Space and Aeronautical Science, University of Tokyo.

NEwSOME, R.W. 1984 Numerical simulation of near-critical and unsteady, subcritical inlet flow. AIAA J. 22 (10), 1375-1379.

PALACIOS, F., et al. 2013 Stanford university unstructured $\left(\mathrm{SU}^{2}\right)$ : an open-source integrated computational environment for multi-physics simulation and design. In 51st AIAA Aerospace Sciences Meeting including the New Horizons Forum and Aerospace Exposition. American Institute of Aeronautics and Astronautics.

RaO, S.M.V. \& KARTHick, S.K. 2019 Studies on the effect of imaging parameters on dynamic mode decomposition of time-resolved schlieren flow images. Aerosp. Sci. Technol. 88, 136-146.

Rodi, P.E., Emami, S. \& TreXler, C.A. 1996 Unsteady pressure behavior in a ramjet/scramjet inlet. J. Propul. Power 12 (3), 486-493.

Soltani, M.R. \& SePahi-Younsi, J. 2016 Buzz cycle description in an axisymmetric mixed-compression air intake. AIAA J. 54 (3), 1040-1053.

SterbentZ, W.H. \& DaVIDS, J. 1955 Amplitude of supersonic diffuser flow pulsations. NACA Tech. Note 3572 . 
TAN, H.-J., SHU, S. \& ZHI-LONG, Y. 2009 Oscillatory flows of rectangular hypersonic inlet unstart caused by downstream mass-flow choking. J. Propul. Power 25, 138-147.

Trapier, S., Duveau, P. \& DeCK, S. 2006 Experimental study of supersonic inlet buzz. AIAA J. 44 (10), 2354-2365.

VAN WIE, D., KwoK, F. \& WALSh, R. 1996 Starting characteristics of supersonic inlets. In 32nd Joint Propulsion Conference and Exhibit, p. 2914.

Wagner, J.L., Yuceil, K.B., Valdivia, A., Clemens, N.T. \& Dolling, D.S. 2009 Experimental investigation of unstart in an inlet/isolator model in Mach 5 flow. AIAA J. 47, 1528-1542.

Zhang, Q.-F., TAN, H.-J., Chen, H., YuAn, Y.-Q. \& ZhANG, Y.-C. 2016 Unstart process of a rectangular hypersonic inlet at different Mach numbers. AIAA J. 54 (12), 3681-3691. 